\title{
Shape discriminations of three-dimensional objects depend on the number and location of bends
}

\author{
DAVID LAWRENCE HALL and ALINDA FRIEDMAN \\ University of Alberta, Edmonton, Alberta, Canada
}

\begin{abstract}
In three experiments, subjects made shape discriminations of three-dimensional objects differing in orientation, number of bends, and location of bends (e.g., the central arm vs. a minor subarm). In general, encoding times at $0^{\circ}$ disparity on both same and different trials were affected by the number of bends, but only after a certain threshold of bends in the objects had been reached (Experiment 1). This effect was not due to the subjects' having to search for matching ends of the objects (Experiment 2). In contrast, rotation rates were influenced by the location of the bends, but not by the number of bends per se (Experiment 3). The results support a representational scheme that is hierarchical, but not necessarily one in which the principal axis of an object is paramount.
\end{abstract}

When observers decide whether two objects shown in different orientations have the same shape, decision times typically increase linearly as a function of the angular disparity between the objects (Metzler \& Shepard, 1974; Shepard \& Metzler, 1971). It is hypothesized that the intercept of this function reflects encoding and decision processes, and the slope reflects the time to transform and compare representations of each stimulus (Cooper \& Shepard, 1973; Just \& Carpenter, 1976, 1985). The main purpose of the present experiments was to determine how the structure of the objects themselves influences encoding and transformation times. In particular, we explore how judgments about an object's shape are influenced by manipulations that should affect the complexity of the object's representation.

In general, the complexity of an object's structure should influence both the time to encode it and the time to transform its representation. Most researchers who have explored the effects of stimulus structure on encoding and transformation times in a mental rotation task have used two-dimensional (2-D) stimuli (Cooper, 1975; Cooper \& Podgorny, 1976; Bethell-Fox \& Shepard,

\footnotetext{
Order of authorship was determined by a coin toss. This research was supported by a grant to the second author by the Natural Sciences and Engineering Research Council of Canada. The first two experiments were conducted in partial fulfillment of the requirements for the MA degree of the first author, and were presented at the meetings of the Canadian Psychological Association and the Canadian Society of Brain, Behavior, and Cognitive Science, Calgary, Alberta, June 1991. We would like to thank Peter Dixon, John Henderson, and Roger Toogood for comments on the thesis, and Vincent Di Lollo for comments and discussion of an earlier version of the manuscript. In addition, the manuscript benefited a great deal from suggestions by Pierre Jolicoeur, Lester Krueger, and two anonymous reviewers. Requests for reprints should be addressed to D. Hall, Department of Psychology, University of Alberta, Edmonton, AB, Canada T6G 2E9.
}

1988; Folk \& Luce, 1987; Hochberg \& Gellman, 1977). Thus, data relevant to the effects of three-dimensional (3-D) structure are sparse. Perhaps as a consequence, some theories of representation, as applied to the mental rotation task, have not yet fully integrated the third dimension (e.g., Tarr \& Pinker, 1990). Yet it is the alleged resemblance between the operations used in the mental rotation task and the operations used to recognize real 3-D objects under transformation that has motivated much of the previous literature (see Shepard \& Cooper, 1982 , for review). Indeed, the kind of structural manipulations that influence performance on a mental rotation task may favor one or another scheme for representing 3-D structure (e.g., Biederman, 1987; Hinton, 1979; Koenderink \& van Doorn, 1979; Kosslyn, 1981; Marr \& Nishihara, 1978; Pylyshyn, 1981; Rock, 1973; Tarr \& Pinker, 1990). In the present experiments, we determine the effect of adding bends to a 3-D stimulus with respect to two such representational schemes: representation by parts, in which all parts are equivalent, and representation by hierarchical structures, in which some parts are more accessible or important than others. The two schemes differ in their predictions about what adding bends to a 3-D object will do to subjects' encoding and transformation times. In particular, when all parts are equivalent, the location of an additional part should be immaterial, but this is not so in a hierarchical representation.

Although Shepard and Cooper (1982) argued that evidence for the effects of stimulus complexity on transformation times was inconclusive, such evidence has been found more recently (Bethell-Fox \& Shepard, 1988; Folk \& Luce, 1987; Yuille \& Steiger, 1982). For example, Bethell-Fox and Shepard found that encoding times as well as transformation and comparison times increased as the number of pieces in 2-D patterns increased, although this effect diminished with practice. Their stim- 
uli were 2 -D patterns of filled-in squares within a $3 \times 3$ matrix, where separated groups of squares were considered to be separate pieces.

Yuille and Steiger (1982) added blocks to 3-D figures that were similar to those used by Shepard and Metzler (1971), and made some featural information redundant and therefore unnecessary for the discrimination task. When some of the subjects were informed of the redundancy, the slope of the reaction time (RT) function was shallower than the slope for the uninformed subjects, leading Yuille and Steiger to argue that the informed subjects mentally rotated a representation of just part of the figure (see also Hochberg \& Gellman, 1977). Like Bethell-Fox and Shepard (1988), Yuille and Steiger concluded that increased figural complexity slowed the rate of mental rotation, but unlike Bethell-Fox and Shepard, they found virtually no effect of figural complexity on encoding time.

Bethell-Fox and Shepard's (1988) and Yuille and Steiger's (1982) findings support the idea that both 2and 3-D stimuli are represented in terms of their parts, that the parts have equal status in the representation, that the representations are rotated piecemeal, and that each additional piece contributes to the overall rotation rate. Additional support for this idea comes from eye-movement data. For example, when Just and Carpenter (1985) monitored people's eye movements as they were solving a cube-rotation task, they found that each face of the cube was mentally rotated in separate episodes. Thus, the extant data suggest that a relatively simple metric for figural complexity, such as the total number of parts in a figure, determines the number of pieces that are represented and processed. This in turn influences the slope, and possibly the intercept, of the RT function (BethellFox \& Shepard, 1988; Just \& Carpenter, 1985).

When parts of a stimulus are separated in space, as they can be in a 2-D matrix (Bethell-Fox \& Shepard, 1988 ) or on the separate faces of a cube (Just \& Carpenter, 1985), then what constitutes a part is straightforward. It is less clear what constitutes a part for many 3-D objects, but for the type of 3-D objects used by Shepard and Metzler (1971), Yuille and Steiger (1982), and others (Friedman \& Hall, 1992; Friedman \& Pilon, in press; Jolicoeur, Regehr, Smith, \& Smith, 1985; Just \& Carpenter, 1976), in which all parts are rectangular solids attached via $90^{\circ}$ junctions, the parsing task might be relatively simple. In particular, several extant theories propose that both bends and curvature in contours are used to parse 3-D objects into parts as well as to compute the orientation of their surfaces and to determine spatial relations (e.g., Biederman, 1987; Hoffman \& Richards, 1984; Jolicoeur, Ullman, \& Mackay, 1986; Ullman, 1984, 1989). For Shepard-Metzler figures, this means that each $90^{\circ}$ junction begins a new piece. Thus, the more junctions or bends in the object, the more "parts" in its representation.

A related alternative to "representation by parts" is "representation by axes." An axis-based representation, in which both major and minor axes of an object are rep- resented by vectors (Marr $\&$ Nishihara, 1978), has been hypothesized as being used for many common 3-D shapes, including the kinds of block-like figures used by Shepard and Metzler (1971; see also Just \& Carpenter, 1976, 1985). It is somewhat difficult to distinguish between representation by parts and representation by vectors for the kinds of 3-D objects we used; indeed, if every bend in the figure signals a new part or a new vector, the two representational schemes are functionally identical. For the present, the important point is that the simplest version of these two representational schemes for 3-D objects assumes that each part of the object is equal in status. The predictions made for performance are therefore straightforward and indistinguishable from predictions for the 2-D case: If the number of bends is used to parse an object into parts/vectors, the more bends in the object, the more parts/vectors that object has in its representation. The time to parse the object is thus a function of the number of bends, which should be reflected in encoding times (i.e., intercepts) on both same and different trials. Similarly, if all parts of an object are independently transformed, transformation and comparison rates (i.e., slopes) will also be a function of the number of bends.

An alternative to the hypothesis that encoding times and rotation rates will increase monotonically is that performance will not be monotonic, because not all parts of an object have equal status in its representation. For example, the representation might be hierarchical (Hinton, 1979; Marr \& Nishihara, 1978; Palmer, 1989), so that an object's principal axis of elongation is represented by a main vector (or by a generalized cylinder enveloping several vectors; Marr \& Nishihara, 1978) that is "higher" in the hierarchy or is more accessible than are the representations of minor axes. It has been shown that the orientation of an object's principal axis (or the orientation of its top, if it is a familiar object) influences shape discriminations (Friedman \& Hall, 1992; Friedman \& Pilon, in press; Humphrey \& Jolicoeur, 1988; Humphreys, 1984; Parsons, 1987). Thus, in addition to orientation, the structure of an object along its principal axis might be more important for shape discriminations than the structure of the object along its minor axes. If so, the location of bends in an object (e.g., main axis vs. minor axes) will be more important for performance than their sheer number.

\section{EXPERIMENT 1}

We had evidence from previous work with a shape discrimination task that objects with more bends produce RT functions with steeper slopes and higher intercepts than objects that are relatively simpler (Friedman \& Hall, 1989; Friedman, Pilon, \& Gabrys, 1988). However, we did not systematically manipulate either the location or the number of bends. In Experiment 1, as a first attempt to manipulate the complexity of 3-D stimuli, we manipulated both the number and the location of the bends. We used figures with two, three, four, and five 
bends, which, therefore, had three, four, five, and six parts/vectors, respectively (see Figure 1). To investigate the location factor, we constructed four conditions: the central arm of the object was either straight or bent, and one of the two subarms was either straight or bent (see Figure 1). Pairs of identical or mirror-image figures were displayed at rotational disparities that ranged from $0^{\circ}$ to $150^{\circ}$, in $30^{\circ}$ increments.

From Figure 1 it can be seen that the simplest objects, on the left side of the figure, have the fewest bends (two), and the objects on the far right have the most (five). The straight-central-arm/bent-subarm and bentcentral-arm/straight-subarm conditions each have three and four bends, respectively. Thus, if the number of bends is the appropriate complexity metric for these sorts of shapes, then depending on which processes are affected, intercepts, slopes, and errors should increase monotonically according to the following order of centralarm/subarm conditions: straight-straight, straight-bent, bent-straight, and bent-bent. In contrast, if all parts of an object do not have equal status in its representation, then we should not see monotonicity in any performance measure as a function of the number of bends. Rather, performance should be a function of the location of the bends.

We were interested in encoding times on both same and different trials. However, Just and Carpenter's (1976, 1985) eye-movement data indicated that subjects differ from trial to trial and from each other in terms of how and when they react to detecting an inconsistency on a different trial; for example, sometimes they terminate and respond immediately and other times they may reprocess the entire trial (Just \& Carpenter, 1985). Thus, different trials represent a mix of strategies, and there is no way to determine reliably when inconsistencies are noticed during the rotation process. It is therefore difficult to interpret the parameters of the RT function (i.e., the intercept and slope) on these trials. To avoid this problem, we included trials for which there was no angular disparity between the shapes $\left(0^{\circ}\right.$ disparity trials), so the observed intercepts on same and different trials were not dependent on the RT function per se. We provide the RT data from different trials in the remaining angular disparity conditions in figures throughout the manuscript for comparative purposes, but do not analyze them (see also Just \& Carpenter, 1985; Metzler \& Shepard, 1974).

From Figure 1, it can also be seen that the number of bends and the location of bends are somewhat confounded. That is, bending the central arm the way we did preserves the axis of elongation of the figure, but necessarily adds two bends to the object, whereas bending the subarm adds only one bend. This means that the bent-central-arm conditions have more bends overall than the bent-subarm conditions. We address this issue in Experiment 3, in which all the objects have an equal number of bends.

In addition to the hypotheses that either the number of bends or their location would predict performance, we considered a third alternative. The direction of the bottom subarm relative to the direction of the top subarm can be used in all four conditions to distinguish figures from their mirror images (see Figure 1). Thus, in all cases, only two parts (the top and bottom subarm) are required to discriminate the shapes from their mirror images. Therefore, according to the relevance criterion suggested by Yuille and Steiger (1982; see also Hochberg \& Gellman, 1977), all conditions should be equally difficult. However, our subjects were not informed of the redundancy, and it will be obvious from the data for all three of the experiments that this alternative can be rejected.

\section{Method}

Subjects. Six female and 6 male subjects were recruited from the University of Alberta Department of Psychology research personnel and graduate students. They were paid $5 \notin$ for every correct response on practice and experimental trials, so it was possible for them to earn up to $\$ 31.20$ (Canadian currency) for participating in the experiment. All had normal or corrected-to-normal vision. None had participated in a previous mental rotation experiment.

Stimuli and Design. Stimuli were constructed using a computer graphics package (AutoCAD 10.0, Autodesk Inc., 1988). The central arm of the figures was either straight or bent, and one subarm was either straight or bent, for a total of four experimental conditions (see Figure 1). Only one shape (and its mirror image) was used in each condition. All the figures were rotated around the same axis about a point in the center of the figure. This axis was oblique relative to the orthogonal planes in the $x, y, z$ coordinate

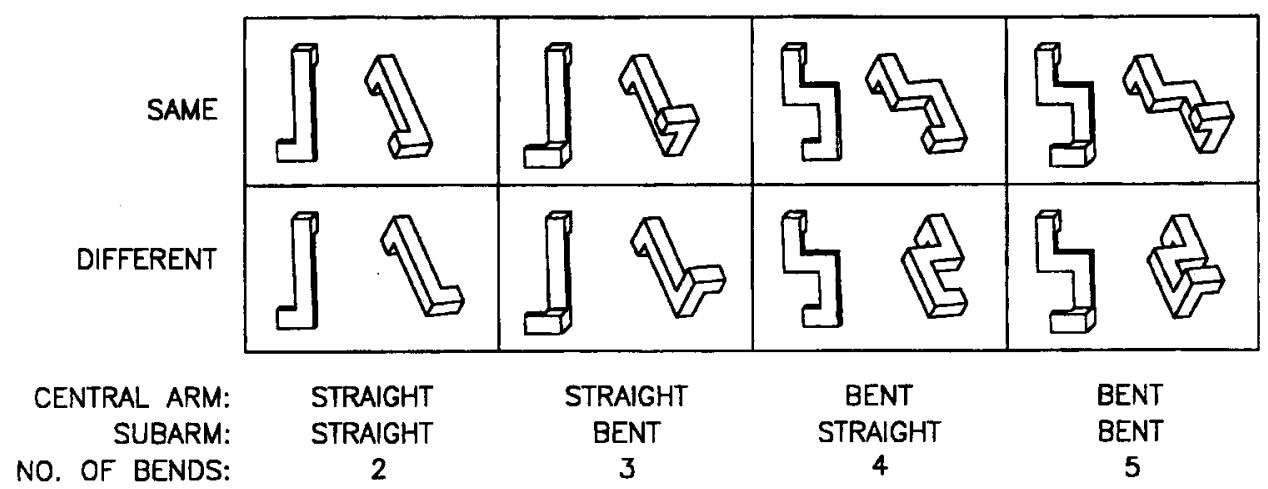

Figure 1. Examples of stimuli from the four conditions of Experiment 1. 
system; it was approximately $35^{\circ}$ from the $x-z$ plane, $12^{\circ}$ from the $x-y$ plane, and $52^{\circ}$ from the $y-z$ plane. Thus, the $x y z$ coordinates of the unit vector of the rotation axis were $-.79, .58, .21$ (see also Parsons, 1987)

The shapes were initially constructed in a vertical position that we will refer to as the starting position. Figures in the starting position for all conditions were the same height. Eleven additional views of each figure were generated by rotating the originals in $30^{\circ}$ increments around the same oblique axis. The same generation procedure was followed using the mirror image of each shape, so that a sequence of 12 views of a given shape and its mirror image were generated - one for each stimulus type (bent or straight central arm $\times$ bent or straight subarm).

From each sequence of 12 views, 12 pairs were formed at each of 6 relative angular disparities $\left(0^{\circ}, 30^{\circ}, 60^{\circ}, 90^{\circ}, 120^{\circ}\right.$, and $\left.150^{\circ}\right)$. This yielded a total of 72 pairs of figures per condition ( 6 relative disparities $\times 12$ pairs each). In addition, if a stimulus and its mirror image are designated as $A$ and $B$, respectively, there are two types of same pairs (AA and $\mathrm{BB}$ ) and two types of different pairs ( $A B$ and $B A$ ), yielding a total of 288 possible stimulus pairs for each of the experimental conditions ( 4 combinations $\times 72$ pairs). A given figure from each pair could be on either the left or the right, yielding $576(288 \times 2)$ total stimuli. The stimuli for a given subject were selected so that, across 4 subjects, all 576 pairs were seen exactly once, and each subject saw the identical pairs in terms of relative angular disparity ( $\mathrm{AA}, \mathrm{BB}, \mathrm{AB}$, or $\mathrm{BA}$ shapes, etc.) in each experimental condition. Corresponding pairs across conditions had approximately the same overall visual angle. Across conditions, the pairs subtended horizontal visual angles ranging between $7.0^{\circ}$ and $11.7^{\circ}$ and vertical angles between $4.3^{\circ}$ and $4.5^{\circ}$.

Stimuli were blocked so that in every 96 trials there was one instance of each AA, BB, AB, BA pair at each of the six relative angular disparities for each of the four central arm $\times$ subarm combinations. The stimuli were randomly presented within these blocks, with the constraint that no more than four consecutive pairs would require the same response. A new random order was generated for each subject.

Apparatus and Procedure. The subjects were seated $87 \mathrm{~cm}$ in front of a Hewlett-Packard 1304a oscilloscope display with a greenish-tint, fast-decay P15 phosphor (decay to $10 \%$ intensity at $2.8 \mu \mathrm{sec}$ after display offset). The resolution was such that lines appeared straight and continuous at all angles. A plotting device developed by Finley (1985) was used to plot the figures on the oscilloscope during each session. A Zenith 159 computer timed the responses and controlled the display devices.

The subjects placed their heads in a chinrest with two laterally placed head stops. The chinrest was used to ensure that their heads were in a comfortable but stable vertical position throughout the trials. A set of touchplates was on the table in front of the subjects. The touchplates consisted of two wooden boards, each with a metal palm plate, a smaller metal finger plate, and a $1.8-\mathrm{cm}-\mathrm{high}$ wooden strip beside each finger plate. The subjects placed one hand on each touchplate so that the heels of the palms rested on one metal plate and the index fingers rested on the wooden strip. The subjects responded by touching their index finger to the smaller metal plate. They used their preferred hand for same responses and the other hand for different responses.

Instructions for the task were read to the subjects. They were then given a short demonstration, in which a pair of figures for one of the conditions was displayed on the screen. The figure on the left was rotated by successive responses on the touchplates, until it was in the same orientation as the figure on the right. This was repeated for eight pairs of figures: one pair of same and one pair of different figures for each of the stimulus conditions. The purpose of the demonstration was to make the concept of mental rotation explicit for the subjects and to give them practice using the touchplates.
After the demonstration, the subjects received 48 practice trials. Practice stimuli were randomly selected pairs from the set of $30^{\circ}, 90^{\circ}$, and $150^{\circ}$ disparities, counterbalanced in the same way as the experimental trials that followed. Each trial began with a short tone, followed $.5 \mathrm{sec}$ later by a fixation cross in the center of the screen for $1 \mathrm{sec}$. Then the cross disappeared and the figures were displayed until the subject responded. If the response was correct, the figures disappeared and the next trial began. If the response was incorrect, two beeps sounded and the figures flashed off and then on again. The figures remained on the screen for another $5 \mathrm{sec}$ so that the subject could review any errors.

Practice trials were followed by two sessions of 288 experimental trials each. The procedure was the same as that followed for practice trials. The subjects received the same auditory feedback that had been given during the practice trials, but they received no visual feedback. After the first session, the subject was given a 5-min rest, and then the second session was given. During practice and experimental trials, the experimenter remained seated behind the subject. At the end of each set of trials, the subjects were told how many trials were correct and how much they had earned. After the second session, they were asked to describe the strategies they had used to perform the task.

\section{Results}

Throughout the present paper, only correct reaction times (RTs) were used to compute means for same and different trials at each disparity and to derive slopes and intercepts. We first examined the RT data for same and different trials at $0^{\circ}$ disparity, because these trials represent the time to encode and decide whether two figures have the same shape when no rotation is required. These times were analyzed in a trial type (same, different) $\times$ central arm (straight, bent) $\times$ subarm (straight, bent) analysis of variance (ANOVA). The data are shown above the $0^{\circ}$ disparity markers in the left and right panels of Figure 2.

Mean RTs at $0^{\circ}$ were faster when the central arm was straight than when it was bent $[1,434$ vs. 2,316 msec; $F(1,11)=36.93, p<.001]$, and were faster when the subarm was straight than when it was bent $[1,690$ vs. $2,060 \mathrm{msec} ; F(1,11)=24.42, p<.001]$. The central arm $\times$ subarm interaction $[F(1,11)=44.85, p<.001]$ and the interactions of trial type $\times$ central arm $[F(1,11)=$ $26.55, p<.001]$, trial type $\times$ subarm $[F(1,11)=15.58$, $p<.01]$, and trial type $\times$ central arm $\times \operatorname{subarm}[F(1,11)=$ $25.60, p<.001]$ were all reliable. The $0^{\circ} \mathrm{RT}$ data for the straight-straight (two bends), straight-bent (three bends), bent-straight (four bends), and bent-bent (five bends) conditions on same trials were $1,213,1,168,1,342$, and $1,374 \mathrm{msec}$, respectively. On different trials, the data were $1,700,1,654,2,504$, and 4,044 $\mathrm{msec}$

It is notable that separate analyses of the same and different trial data showed that the main-effect advantage for the straight-central-arm condition was reliable for both trial types $[F(1,11)=12.20, p<.005$, and $F(1,11)=$ $32.56, p<.001$, respectively], but that the central arm $\times$ subarm interaction was only reliable on different trials $[F(1,11)=38.23, p<.001]$. Contrasts on the data for different trials showed that the $804-\mathrm{msec}$ increase, due to a bend in the central arm (straight-straight vs. bent--straight), was reliable $[F(1,11)=13.30, p<.01]$. 


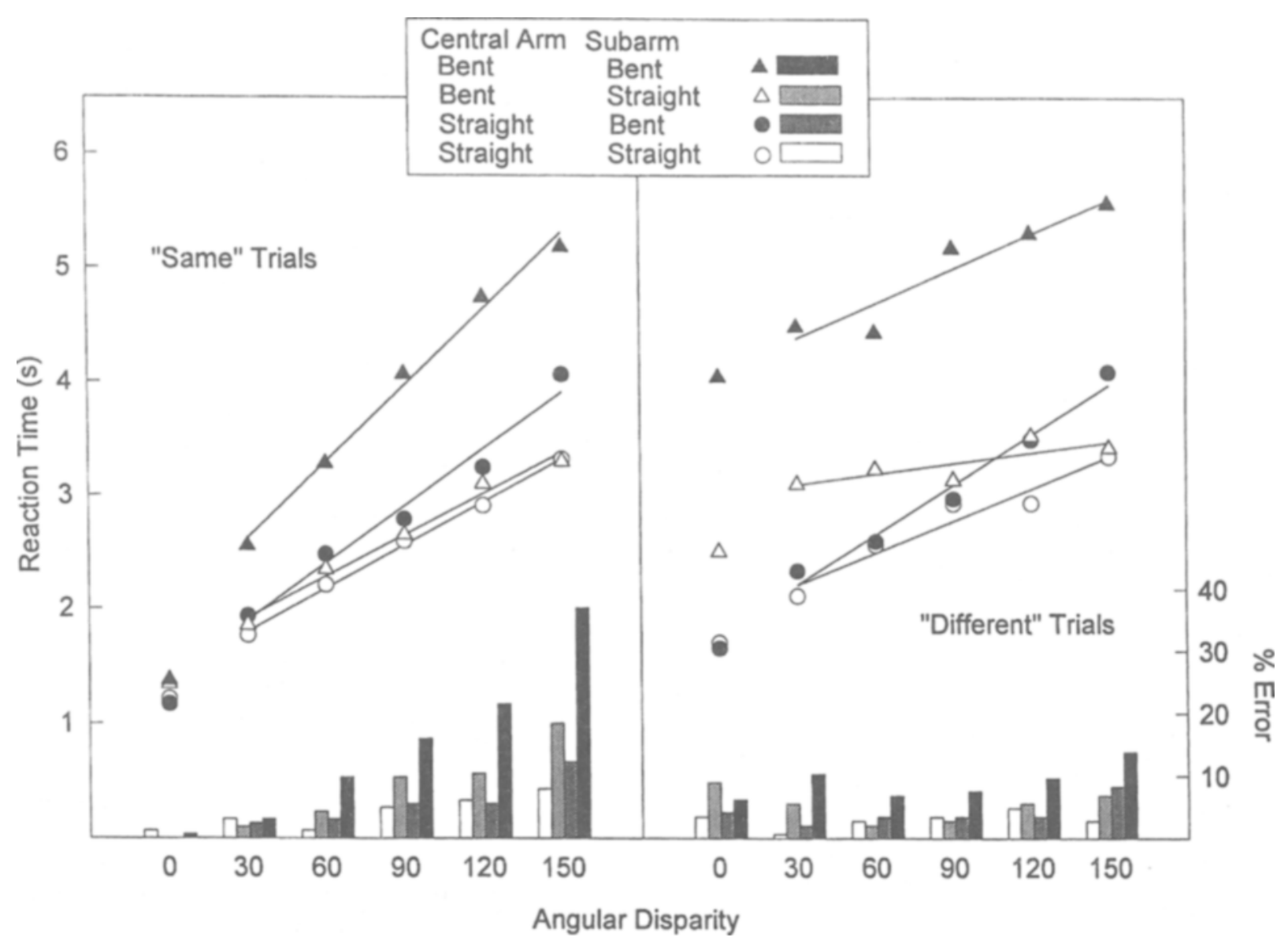

Figure 2. Mean RTs and errors from same (left panel) and different (right panel) trials in Experiment 1. The RTs are from correct trials only. Regression lines are computed excluding RTs on $0^{\circ}$ disparity trials.

When there was already a bend in the central arm (bent-straight condition), the addition of a bend to the subarm (bent-bent condition) also produced a reliable 1,540 -msec increase in RT $[F(1,11)=28.45, p<.001]$. In general, then, the RTs at $0^{\circ}$ on both same and different trials did not increase monotonically with the number of bends; thus they are not compatible with a strict number-of-bends hypothesis, but they are compatible with the notion that it takes longer to "set up" the representation when the central arm is bent.

Responses for same trials were always given with the preferred hand, so the main effect of trial type in favor of faster RTs at $0^{\circ}$ on same trials [1,274 and $2,476 \mathrm{msec}$ for same vs. different trials, respectively; $F(1,11)=28.89$, $p<.001$ ] is most likely due, at least in part, to the responding hand. However, the other main effects and the interactions with trial type discussed above are not easily accounted for in terms of this factor.

Analyses of the overall RTs on same trials as a function of condition and disparity allow us to examine the slopes of the functions and, hence, the influence of the number and location of bends on the rate of the rotation process. Mean RTs on same trials were analyzed in a re- peated measures ANOVA, in which the factors were central arm (straight, bent), subarm (straight, bent), and disparity $\left(0^{\circ}, 30^{\circ}, 60^{\circ}, 90^{\circ}, 120^{\circ}\right.$, or $\left.150^{\circ}\right)$; the latter was treated as a quantitative factor. These data are shown in the left panel of Figure 2.

The main effects of central arm $[F(1,11)=29.66, p<$ $.001]$ and subarm $[F(1,11)=61.96, p<.001]$ were reliable, and their interaction $[F(1,11)=21.76, p<.001]$ was also reliable. The means for the straight-straight, straight-bent, bent-straight, and bent-bent conditions, respectively, were $2,335,2,614,2,433$, and $3,532 \mathrm{msec}$. Thus, overall RTs also did not increase monotonically as a function of the number of bends.

The main effect of disparity was reliable $[F(5,55)=$ $55.68, p<.001]$, and $99.0 \%$ of the variance was accounted for by the linear trend $[F(1,11)=85.04, p<$ $.001]$. The central arm $\times$ angular disparity interaction was also reliable $[F(5,55)=2.41, p<.05]$, and $64.7 \%$ of the variance in the interaction was accounted for by the linear (central arm) $\times$ linear (disparity) component $[F(1,11)=6.04, p<.032]$. This contrast indicates that the slope of the RT function was reliably steeper when the central arm was bent $(19.2 \mathrm{msec} / \mathrm{deg})$ than when it 
was straight $(15.8 \mathrm{msec} / \mathrm{deg})$. Similarly, the subarm $\times$ angular disparity interaction was reliable $[F(5,55)=$ $20.00, p<.001]$, and $97.6 \%$ of the variance was accounted for by the linear (subarm) $\times$ linear (disparity) component $[F(1,11)=80.37, p<.001]$. The slopes were 21.5 and $13.4 \mathrm{msec} / \mathrm{deg}$ when the subarm was bent and straight, respectively. There was also a reliable interaction of cubic (disparity) $\times$ shape of subarm $[F(1,11)=$ $7.71, p<.025]$, which was largely uninterpretable. Finally, the central arm $\times$ subarm $\times$ disparity interaction was reliable $[F(5,55)=3.22, p<.05]$, with $82.9 \%$ of the variance accounted for by the interaction among the linear components $[F(1,11)=7.76, p<.02]$. Slopes for the straight-straight, straight-bent, bent-straight, and bentbent conditions were $13.7,17.9,13.2$, and $25.1 \mathrm{msec} / \mathrm{deg}$, respectively. These conditions represent figures with two, three, four, and five bends, respectively, so it can be seen that rotation rates do not increase monotonically with the number of bends.

When we examined the left panel of Figure 2, we thought it possible that RTs from the $0^{\circ}$ disparity trials were spuriously low. When we reanalyzed the same trial data, omitting the $0^{\circ}$ disparity trials, all the main effects remained reliable, as did the interaction between central arm and subarm $[F(1,11)=22.16, p<.001]$. However, the only reliable interaction with the disparity factor was then the subarm $\times$ disparity interaction $[F(4,44)=10.73$, $p<.001]$ and its linear (subarm) $\times$ linear (disparity) component $[F(1,11)=41.43, p<.001]$. The slope of the function was then $19.6 \mathrm{msec} / \mathrm{deg}$ when the subarm was bent and $12.4 \mathrm{msec} / \mathrm{deg}$ when it was straight. Eliminating the $0^{\circ}$ disparity trials eliminated the central arm $\times$ disparity interaction and its linear component, the central arm $\times$ subarm $\times$ disparity interaction and its linear component, and the cubic (disparity) $\times$ subarm interaction. The new slopes for the straight-straight (two bends), straight-bent (three bends), bent-straight (four bends), and bent-bent (five bends) conditions on same trials were $12.7,16.8,12.2$, and $22.4 \mathrm{msec} / \mathrm{deg}$, respectively, which was the same ordinal relation as that obtained when RTs at $0^{\circ}$ were included in the analysis. Nevertheless, the regression lines displayed in Figures 2, 4 , and 6 are the ones that exclude the RTs at $0^{\circ}$.

Importantly, intercepts computed from the RT function with $0^{\circ}$ disparity trials excluded also showed the same ordinal relations as did the RTs at $0^{\circ}$. Computed intercepts on same trials for the straight-straight, straightbent, bent-straight, and bent-bent conditions were 1,416 , $1,394,1,557$, and $1,948 \mathrm{msec}$, respectively; on different trials they were $1,926,1,773,3,002$, and 4,085 msec.

Errors for same and different trials were analyzed in a trial type $\times$ central arm $\times$ subarm $\times$ disparity ANOVA and are shown in the bar graphs of Figure 2. According to the noisy-operator theory proposed by Krueger (1978, 1985), an increase in complexity (or difficulty) in a discrimination task increases the likelihood of spurious mismatches on same trials, which then creates a bias toward making a different response. In our task, assuming that subjects compare a pair of shapes by matching each corresponding bend, each bend adds a potential source of error in the matching process. Actual mismatches will be detected if corresponding bends are from a different pair. Spurious mismatches will be detected if corresponding bends from a same pair are misaligned (e.g., at large angles of disparity) or if, due to "noise," such bends are misrepresented. Thus, a larger number of spurious mismatches might be expected for same pairs at larger angles of disparity and for figures with a large number of bends.

In accord with these expectations, more errors were made on same trials than on different trials [ $8.8 \mathrm{vs.} 5.4 \%$; $F(1,11)=9.21, p<.05]$. The subjects made more errors when the central arm was bent than when it was straight $[F(1,11)=11.54, p<.01]$, and they made more errors when the subarm was bent than when it was straight $[F(1,11)=64.97, p<.001]$. The central arm $\times$ subarm interaction was also reliable $[F(1,11)=6.37, p<.05]$. The mean percentage of errors for the straight-straight, straight-bent, bent-straight, and bent-bent conditions were $3.8 \%, 6.9 \%, 4.8 \%$, and $12.8 \%$, respectively. As was also predicted by the noisy-operator theory, the difference between same and different trials increased as a function of disparity $[F(1,11)=13.67, p<.001]$. The central arm $\times$ angular disparity interaction $[F(5,55)=$ $7.71, p<.001]$, the subarm $\times$ angular disparity interaction $[F(5,55)=7.00, p<.001]$, and the three-way interaction of trial type $\times$ subarm $\times$ disparity $[F(1,11)=$ $8.25, p<.001]$ were all reliable. In general, errors increased with disparity on same trials, and more so for the difficult conditions. On different trials, errors did not vary as a function of condition.

It should be recalled that different stimuli took considerably longer to encode than same stimuli for the RT at $0^{\circ}$ data; the trial type $\times$ central arm $\times$ subarm interaction indicated that this was especially true for the bentbent condition. The encoding-time differences on different trials are also consistent with the noisy-operator theory (Krueger, 1978, 1985). An increase in spurious mismatches leads to an increased likelihood, for our task, of reencoding the figures and reprocessing them on at least some of the different trials, which will increase the RTs at $0^{\circ}$. Convergent evidence for such reencoding and rechecking in a mental rotation task comes from Just and Carpenter's $(1976,1985)$ eye-movement data.

\section{Discussion}

First, it is clear that none of the measures increased monotonically with the number of bends in the figure, whereas there were effects of bend location on RTs at $0^{\circ}$ for same and different trials, overall RTs and slopes on same trials, and errors.

Second, the effect of bend location on rotation rates for same trials was ambiguous. When RTs on $0^{\circ}$ disparity trials were included in the function, the bent central arm and the bent subarm produced steeper slopes than the corresponding straight conditions, but when RTs at $0^{\circ}$ were excluded, only the bend in the subarm made a difference to rotation rates. What is clear is that the loca- 
tion of the bends influences transformation rates (and certainly, overall processing times), but the way that it does so is opposite to a location hypothesis, which targets the structure of the central arm as the source of difficulty.

Finally, RTs in the $0^{\circ}$ disparity condition were influenced by the shape of the central arm on both same and different trials, but the shape of the subarm only influenced RTs at $0^{\circ}$ on the different trials. Because of this, we would like to conclude that the complexity of the subarm's structure influenced encoding time only when the subjects were engaging in some sort of rechecking process on different trials. However, there is another possibility. Just and Carpenter (1976, 1985; see also Metzler \& Shepard, 1974) showed that subjects initially search for corresponding ends of two figures that make up a pair before beginning a rotation episode. It is possible that, for our stimuli, corresponding ends are harder to find when the central arm is bent than when it is straight. Therefore, the effect of central-arm structure on the intercept might be due to the fact that it makes searching for corresponding ends more difficult, rather than to difficulties inherent in encoding the structure per se.

\section{EXPERIMENT 2}

One way to test the two interpretations of the RT at $0^{\circ}$ data is to make the ends of the stimuli more easily identifiable. For example, if the corresponding ends of two figures are color coded, they should be easier to find and match (e.g., Metzler \& Shepard, 1974, Experiment 2), and any difficulty in finding corresponding ends attributable to the bent central arm should be attenuated. On the other hand, if setting up the representation for bentcentral-arm figures takes longer than for straight-centralarm figures, coloring the ends will not eliminate this source of difficulty. Therefore, in Experiment 2, we compared performance with pairs of figures whose corresponding bottom subarms either were or were not color coded.

\section{Method}

Subjects. Six female and 6 male subjects were recruited from the University of Alberta Department of Psychology research per- sonnel and graduate students. They were paid $5 \not$ for every correct response on practice and experimental trials, so it was possible for them to earn up to $\$ 31.20$ (Canadian currency) for participating in the experiment. All had normal or corrected-to-normal vision. None had participated in a previous mental rotation experiment.

Stimuli and Design. The figures were those that had been used in the straight-bent and bent-bent conditions of Experiment 1. Thus, all the figures had a bent subarm and either a straight or bent central arm. The stimuli were displayed as white line drawings on a dark screen. They subtended approximately the same visual angles as the stimuli in Experiment 1. The edges of the bottom subarm either were the same color as the rest of the stimulus (white) or were colored a bright green (see Figure 3 ). Thus, there were four combinations of central arm (straight, bent) $\times$ color of subarm (colored, neutral) conditions. Green was selected over the range of available colors because it seemed to be the most noticeable, and it seemed to display the same brightness as the rest of the figure. The figures were the same size, were displayed at the same distance, and were rotated around the same oblique axis that had been used in Experiment 1 . The remaining within- and between-subjects factors were counterbalanced as they had been in Experiment 1 .

Apparatus and Procedure. The instructions and procedure were the same as those used in Experiment 1, with the following exceptions. First, the researcher left the experiment room prior to the experimental trials. Second, each subject was instructed to initiate a given set of trials by tapping a touchplate once. Third, to display the colored subarms, we showed the stimuli with an NEC 3D Multisync monitor driven by a Paradise VGA video board, rather than on the oscilloscope that was used in Experiment 1.

\section{Results}

Results from the analysis of RTs at $0^{\circ}$ replicated those from Experiment 1; they are shown above the $0^{\circ}$ disparity markers in Figure 4. As expected, RTs for same trials were faster than those for different trials $[F(1,11)=$ $34.41, p<.001]$, and figures with a straight central arm were encoded more quickly than figures with a bent central $\operatorname{arm}[F(1,11)=24.17, p<.001]$. Figures that were color coded were faster to encode than figures with neutral subarms $[F(1,11)=24.65, p<.001]$. The trial type $\times$ central arm interaction was reliable $[F(1,11)=12.08$, $p<.01]$, but no other interactions were reliable. Mean RTs at $0^{\circ}$ on same trials for the straight-colored, straight-neutral, bent-colored, and bent-neutral conditions were $1,087,1,174,1,271$, and $1,407 \mathrm{msec}$, respectively; on different trials they were $1,465,1,599,2,626$,

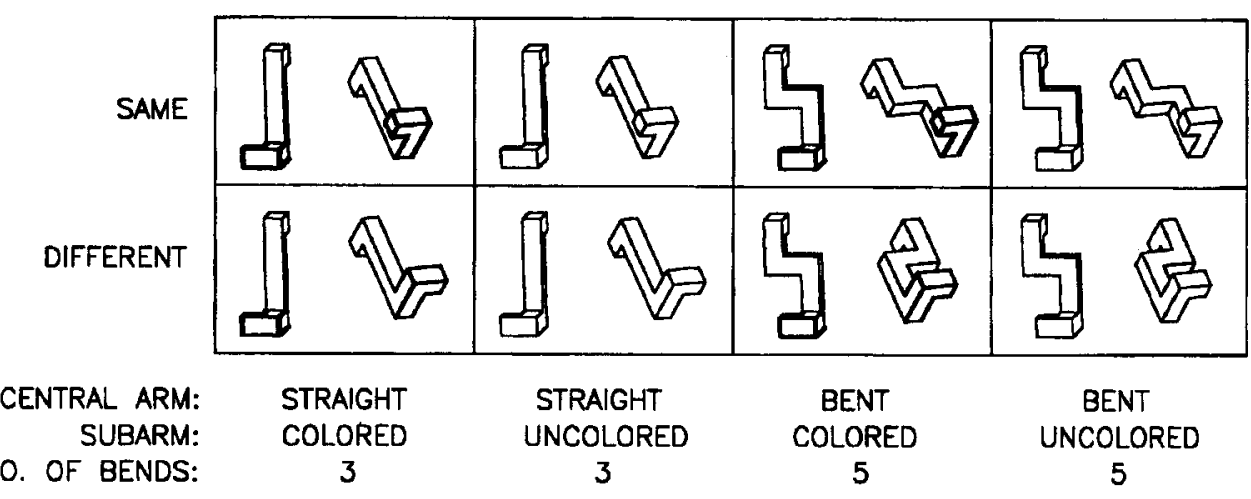

Figure 3. Examples of stimuli from the four conditions of Experiment 2. The thickened lines represent the parts of the stimuli that were colored green in the experimental displays. They appear thicker in the figure, but all the lines were the same thickness in the actual display. 
and 3,022 msec. Thus, the data from RTs at $0^{\circ}$ indicate that color coding attenuated, but did not eliminate, the effect of number of bends in the central arm on encoding, reencoding, and rechecking times.

Mean RTs for same trials were analyzed in a threefactor repeated measures ANOVA, in which withinsubjects factors were shape of the central arm (straight, bent), color of the subarms (colored, neutral), and angular disparity $\left(0^{\circ}, 30^{\circ}, 60^{\circ}, 90^{\circ}, 120^{\circ}\right.$, or $\left.150^{\circ}\right)$. These data are shown in the left panel of Figure 4; the right panel of Figure 4 shows the data on different trials.

Overall same RTs were, again, faster when the central arm was straight than when it was bent $[2,302$ vs. $3,009$ msec; $F(1,11)=51.20, p<.001]$. In addition, color-coded subarms produced faster RTs than neutral subarms $[2,460$ vs. $2,851 \mathrm{msec} ; F(1,11)=27.80, p<$ $.001]$. The two-way interaction was not reliable. Therefore, although color coding the subarms decreased overall RTs, presumably because the corresponding parts of the figures became easier to find and keep track of, it did not eliminate the influence on overall processing time of structural complexity along the central arm of the figures.
Mean RTs increased with disparity $[F(5,55)=53.31$, $p<.001]$, and $99.9 \%$ of the variance was accounted for by the linear trend $[F(1,11)=64.33, p<.001]$. As in Experiment 1 , the central arm $\times$ disparity interaction was reliable $[F(5,55)=2.52, p<.05]$, and $87.3 \%$ of the variance in the interaction was accounted for by the linear (central arm) $\times$ linear (disparity) component $[F(1,11)=9.05, p<.05]$. The slope of the RT function when the central arm was straight was $15.9 \mathrm{msec} / \mathrm{deg}$, and it was $20.6 \mathrm{msec} / \mathrm{deg}$ when the central arm was bent. The color $X$ disparity interaction was unreliable, but the central arm $\times$ color $X$ angular disparity interaction was reliable $[F(5,55)=3.22, p<.05]$. However, the linear component of the interaction was unreliable, indicating that the slopes did not differ among the four conditions.

As in Experiment 1, we reanalyzed the overall RT data on same trials, excluding the $0^{\circ}$ disparity trials, and once again we obtained the same reliable effects, except for the central arm $\times$ angular disparity interaction, its linear component, and the triple interaction. The new slope, when the central arm was straight, was $16.0 \mathrm{msec} / \mathrm{deg}$. The slope was $19.4 \mathrm{msec} / \mathrm{deg}$ when it was bent. Intercepts computed with $0^{\circ}$ excluded on same trials were

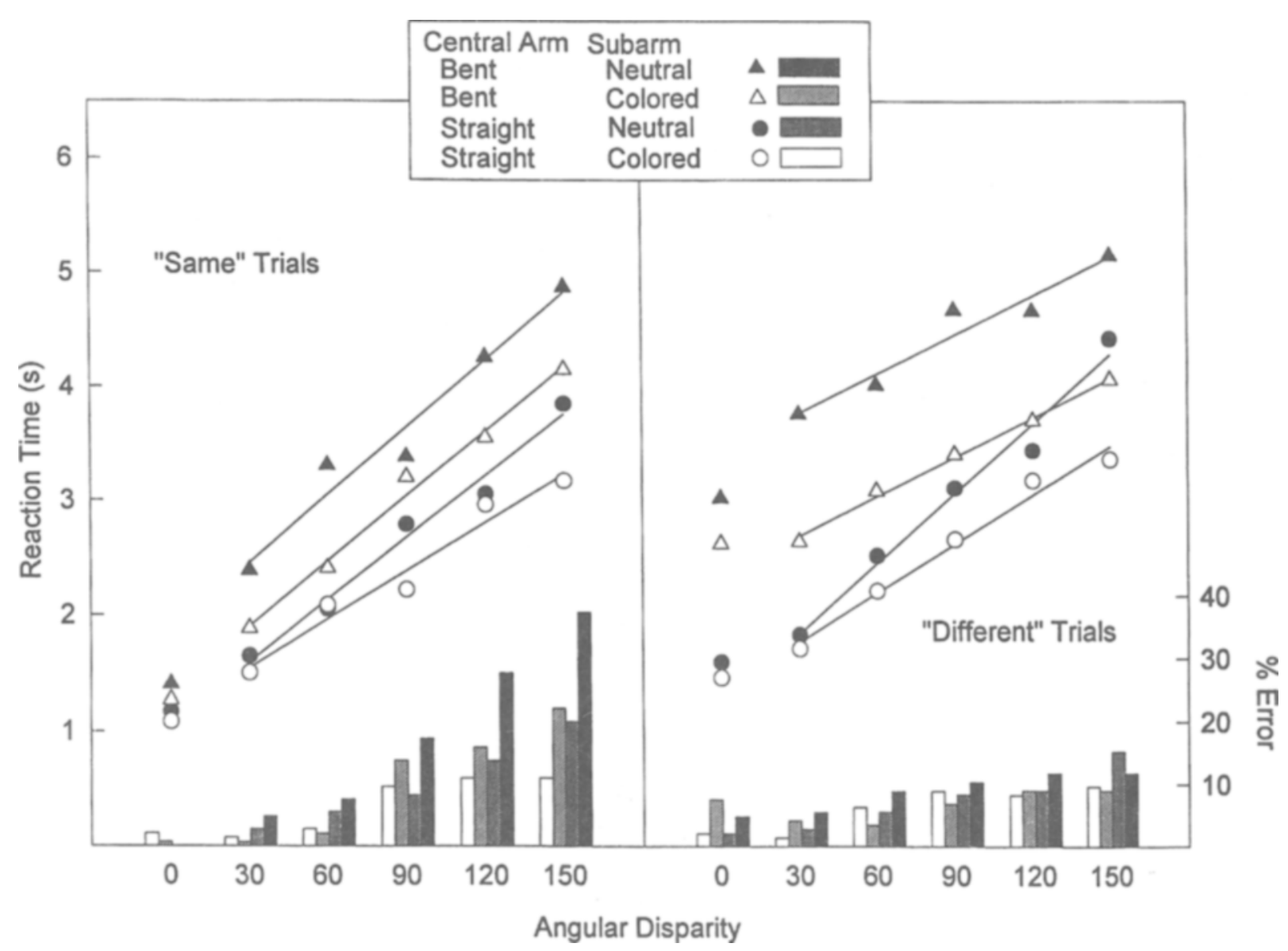

Figure 4. Mean RTs and errors from same (left panel) and different (right panel) trials in Experiment 2. RTs are from correct trials only. Regression lines are computed excluding RTs on $0^{\circ}$ disparity trials. 
$1,129,1,059,1,340$, and $1,865 \mathrm{msec}$, respectively, for the straight-colored, straight-neutral, bent-colored, and bent-neutral conditions. For different trials, they were $1,349,1,233,2,342$, and $3,428 \mathrm{msec}$. Thus, encoding times were still shorter for straight-central-arm figures than they were for bent-central-arm figures, irrespective of color coding.

Errors were analyzed in a trial type $x$ central arm $\times$ color $X$ angular disparity ANOVA; they are shown by the bars at the bottom of Figure 4. More errors were produced on same trials than on different trials $[10.0 \%$ vs. $7.2 \% ; F(1,11)=5.41, p<.05]$. As was expected from the noisy-operator theory (Krueger, 1978, 1985), there were reliable main effects of central arm $[F(1,11)=$ $19.47, p<.001]$, color $[F(1,11)=10.74, p<.01]$, and angular disparity $[F(5,55)=28.11, p<.001]$. The trial type $\times$ disparity interaction was reliable $[F(5,55)=$ $7.72, p<.001]$. The interactions of central arm $\times$ angular disparity $[F(5,55)=3.80, p<.01]$, color $\times$ angular disparity $[F(5,55)=2.53, p<.05]$, and trial type $\times$ color $\times$ angular disparity $[F(5,55)=7.50, p<.001]$ were also reliable. Color served to reduce errors on same trials, especially at the larger angles of disparity. On different trials, the advantage of color was attenuated.

\section{Discussion}

First, as in Experiment 1, the RT at $0^{\circ}$ data, as well as the computed intercepts without RTs at $0^{\circ}$, showed clear effects of central-arm shape on encoding times for both same and different trials. Thus, a bend in the central arm influenced encoding times, even when the ends of the figures were color coded to make them easier to match.

Second, the effect of bend location on the time to transform and compare the stimuli was again ambiguous: With RTs from the $0^{\circ}$ disparity trials included, slopes for the figures with bent central arms were larger than those for the figures with straight central arms. With the $0^{\circ}$ disparity trials removed, however, slopes were not reliably different across the two conditions.

\section{EXPERIMENT 3}

As noted in the introduction, a bend in the central arm adds two bends to the figure, whereas a bend in the subarm adds only one. Therefore, figures with a bend in the central arm but a straight subarm had a greater number of bends altogether than figures with a straight central arm and a bent subarm. If the effect of the bend in the central arm on RTs at $0^{\circ}$ in Experiments 1 and 2 was due to the location of bends, then, if the number of bends was controlled, figures with a bent central arm should have a higher intercept than figures with the same number of bends but with a straight central arm. A similar argument can be made with respect to slopes. Thus, in Experiment 3 , we used figures in which the location of bends was manipulated but the number of bends was kept the same. In this way, we were able to test unambiguously the effect of bend location on encoding times and rotation rates.

\section{Method}

Subjects. Six female and 6 male subjects were recruited from the University of Alberta Department of Psychology research personnel and graduate students. They were paid $5 \notin$ for every correct response on practice and experimental trials. All had normal or corrected-to-normal vision. None had participated in a previous mental rotation experiment.

Stimuli and Design. The figures were made by using the procedure that had been used in Experiment 1. In one condition, the figure had a straight central arm and a bend in each subarm. In the other condition, the figure had straight subarms and a bend in the central arm. Thus, each figure had the same total number of bends (four), but the location of the bends was different (see Figure 5). Display distance and the rotation axis were the same as those that had been used in Experiments 1 and 2. Across conditions, the pairs of figures subtended horizontal visual angles that ranged between $8.0^{\circ}$ and $11.6^{\circ}$ and vertical angles that ranged between $4.0^{\circ}$ and $4.6^{\circ}$. Because the error rates were so high for the complex figures at $150^{\circ}$ in Experiments 1 and 2, we did not use $150^{\circ}$ disparity in Experiment 3.

When we looked at the new figures in each rotation position, it appeared that the bent-central-arm figure in one particular position $\left(150^{\circ}\right.$ from upright) was almost an orthographic projection (i.e.,

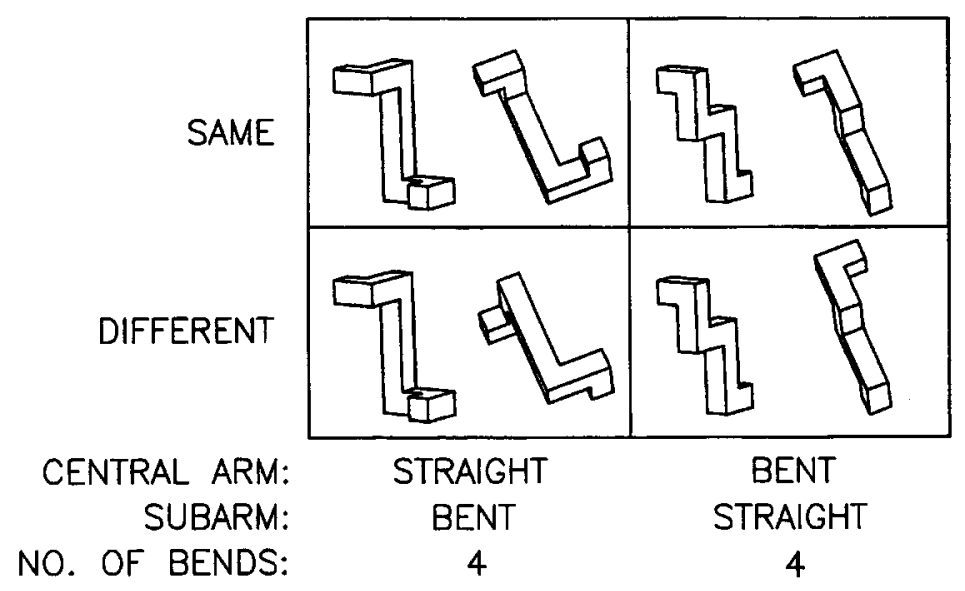

Figure 5. Examples of stimuli from the two conditions of Experiment 3. 
"straight-on" view); therefore, it was difficult to determine the structure of that particular figure in that position relative to all the other positions. Consequently, to ensure that the stimulus set would be balanced across conditions, we did not use that figure/position combination in any stimulus condition for Experiment 3 .

Because one figure position was not used, and because that figure could be on the left or right of a given pair, any particular angular disparity could be produced by a total of 10 (rather than 12) possible pairs of figures for disparities $30^{\circ}$ through $120^{\circ}$, and by a total of 11 (out of 12 ) for $0^{\circ}$ disparity pairs. Trials were blocked in such a way that each block had one figure position (condition, relative disparity, same/different, left-figure-A-or-B) combination. The four remaining $0^{\circ}$ disparity pairs ( $A A, A B, B A, B B$ ) for each condition were randomly assigned to trials in blocks that were randomly selected without replacement (with the constraint that there would be no more than four same or four different trials in a row). Thus, there were 10 blocks of 40 trials, plus 8 trials of $0^{\circ}$ disparity, for a total of 408 experimental trials. A new random order was generated for each subject.

Apparatus and Procedure. The instructions, apparatus, and procedure were the same as those used in Experiment 1.

\section{Results}

Once again, RTs at $0^{\circ}$ on same trials were faster than RTs on different trials $[F(1,11)=23.54, p<.001]$. The main effect of condition $[F(1,11)=11.49, p<.01]$ and the interaction of trial type $\times$ condition $[F(1,11)=10.28$, $p<.01]$ were reliable. For same trials, RTs at $0^{\circ}$ were 1,203 and $1,313 \mathrm{msec}$ for the bent-central-arm and the bent-subarm conditions, respectively. For different trials, the RTs at $0^{\circ}$ were 2,107 and $4,205 \mathrm{msec}$. These data are shown above the $0^{\circ}$ markers in Figure 6 . Although the differences between the conditions were in the same direction, only the one for different trials was reliable $[F(1,11)=10.96, p<.01]$. This difference in $\mathrm{RT}$ at $0^{\circ}$ between conditions is not predicted by the number-ofbends hypothesis, because the number of bends was equal in both conditions. However, the obtained difference contradicts a location hypothesis, which proposes that the complexity of an object along its principal axis is the only important factor.

Overall RTs for same trials were analyzed in a stimulus condition $\times$ disparity $\left(0^{\circ}, 30^{\circ}, 60^{\circ}, 90^{\circ}, 120^{\circ}\right)$ repeated measures ANOVA. These data are shown in the left panel of Figure 6 . The bent-central-arm figures produced shorter RTs than the bent-subarm figures [2,551 vs. $3,311 \mathrm{msec} ; F(1,11)=16.02, p<.01]$. The main ef-

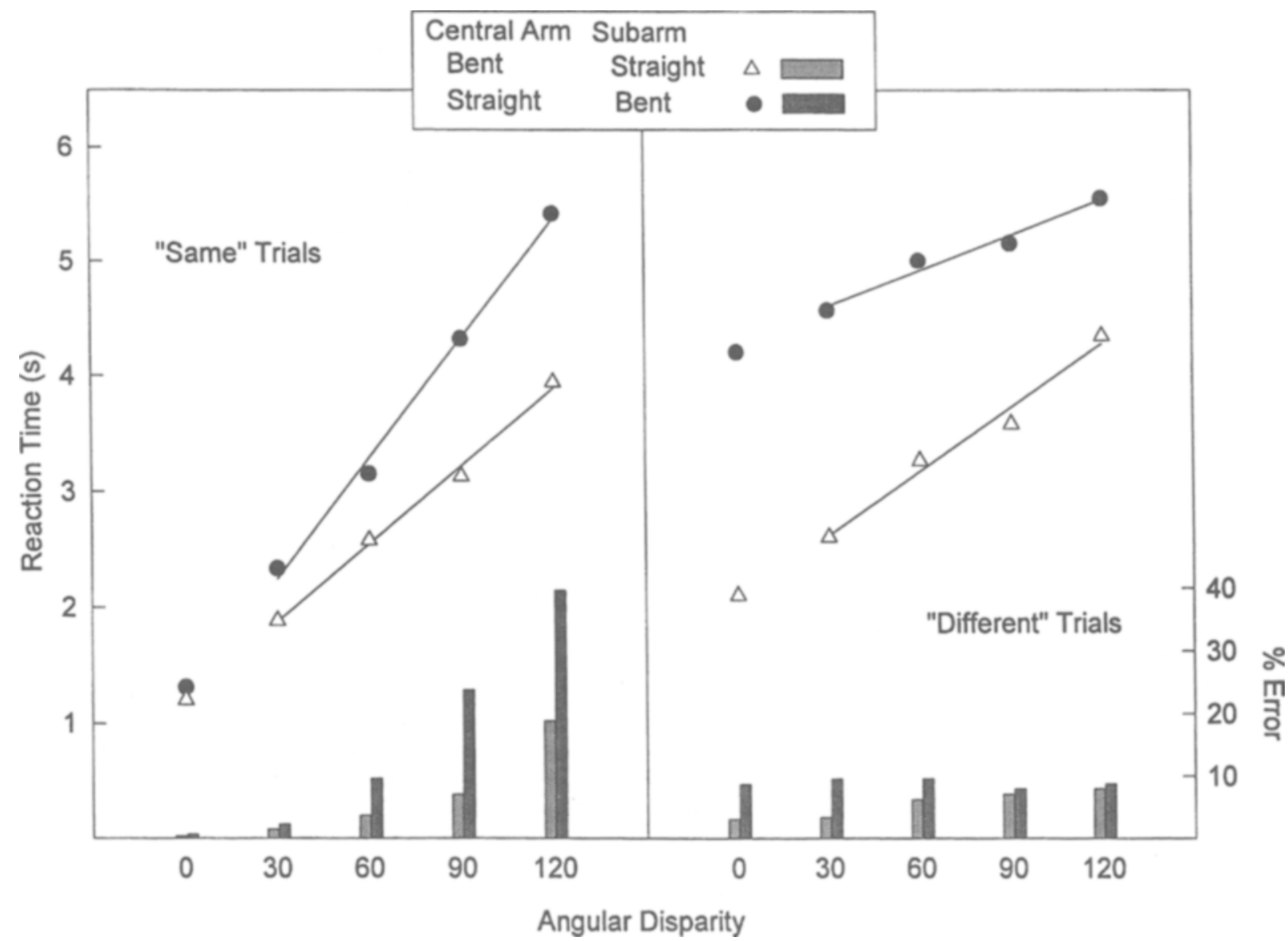

Figure 6. Mean RTs and errors from same (left panel) and different (right panel) trials in Experiment 3. The RTs are from correct trials only. Regression lines are computed excluding RTs on $0^{\circ}$ disparity trials. 
fect of disparity was reliable $[F(4,44)=36.46, p<$ $.001]$, and $99.8 \%$ of the variance was due to the linear trend $[F(1,11)=42.42, p<.001]$. The condition $\times$ disparity interaction was also reliable $[F(4,44)=6.33, p<$ $.001]$, and $96.3 \%$ of the variance was due to the linear component of the disparity factor $[F(1,11)=10.27$, $p<.01]$. The slopes on bent-central-arm trials were $22.4 \mathrm{msec} / \mathrm{deg}$, and those on bent-subarm trials were $34.0 \mathrm{msec} / \mathrm{deg}$.

We reanalyzed the overall RT data on same trials, excluding RTs for $0^{\circ}$ disparity; essentially, everything was the same as it had been when the trials were included. The new slopes were $22.4 \mathrm{msec} / \mathrm{deg}$ in the bent-centralarm condition and they were $34.7 \mathrm{msec} / \mathrm{deg}$ in the bentsubarm condition. In addition, when RTs at $0^{\circ}$ were excluded, the new intercepts were consistent with the notion that initial encoding time was not a function of bend location, because intercepts on same trials were identical for both conditions $(1,208 \mathrm{msec})$. For different trials, the new intercepts were $2,070 \mathrm{msec}$ for the bentcentral-arm condition and $4,295 \mathrm{msec}$ for the bentsubarm condition.

Errors were analyzed in a trial type $\times$ condition $\times$ angular disparity ANOVA. More errors were produced in the bent-subarm condition than in the bent-central-arm condition $[13.0 \%$ vs. $6.3 \% ; F(1,11)=21.47, p<.001]$. Errors increased as a function of angular disparity $[F(4,44)=49.54, p<.001]$, and the condition $\times$ angular disparity interaction was reliable $[F(4,44)=5.54$, $p<.001]$. Again, as posited by the noisy-operator theory (Krueger, 1978), more errors were produced on same trials than on different trials $[12.1 \%$ vs. $7.2 \%$; $F(1,11)=15.09, p<.01]$. As is evident from the bars in Figure 6, the difference between conditions for errors on same trials increased as a function of angular disparity, but errors on different trials remained relatively flat. This is corroborated by the reliable trial type $X$ disparity interaction $[F(4,44)=26.05, p<.001]$, as well as by the three-way interaction $[F(4,44)=12.75, p<.001]$.

\section{Discussion}

The data from Experiment 3 allow us to reject unambiguously the hypothesis that the number of bends per se played a causal role in any performance measure, because the stimuli in both conditions had an equal number of bends. We may thus interpret the data in favor of the location-of-bends hypothesis and, by inference, in favor of a hierarchical representation. However, we must reject a hierarchical representation that targets the shape of the object along its principal axis as a source of transformation difficulty, because rotation rates increased in Experiment 3 only as a function of the presence of bends in the subarms. Thus, the original hypothesis, that a bend in the central arm would in itself make the representation more difficult to transform, is contradicted by the data.

Experiment 3 further showed that encoding times were unaffected by the location of the bends when the number of bends was equated, indicating that the differences in encoding times obtained in Experiments 1 and
2 were also attributable to the number of bends. But the effect of sheer number of bends on encoding times is not straightforward because, although encoding times increased with the number of bends in Experiment 1, the increase was not monotonic. And although Experiment 2 showed that some encoding time is spent looking for matching ends, not all of it was; even in the color-coded conditions, the figures with a bent central arm were encoded more slowly than the figures with a straight central arm.

Because bending the central arm of our objects resulted in more bends overall than bending the subarm, the encoding time differences found in the first two studies may have reflected a "threshold" of difficulty. A parsimonious explanation for the RTs at $0^{\circ}$ across all three studies is that encoding time becomes slower as a function of the number of bends, once a particular threshold is reached. In addition, RTs for $0^{\circ}$ different trials in all three experiments reflect additional rechecking and reprocessing time in the more difficult conditions (Just \& Carpenter, 1985; Krueger, 1978, 1985).

The RT and error data shown in Figure 6 (as well as in Figures 2 and 4) suggest that, owing to the intermixing of easy and difficult trials, the subjects relied on a compromise criterion (Krueger, 1985) that specified a moderate or midlevel degree of perceived difference as the boundary (rechecking) region between responding "same" and "different." This criterion produced a bias toward "same" responses on the easy trials at $0^{\circ}$, for which the spuriously perceived differences on same trials presumably were quite small, resulting in a large fastsame effect and more false-same errors. The compromise criterion also produced a bias toward "different" responses (little or no fast-same effect and more falsedifferent errors) on the difficult trials at $120^{\circ}$, for which the spuriously perceived differences on same trials presumably were quite large (Krueger, 1985).

In sum, the locus of the complexity effect on encoding times seems to be in the number of bends (or parts) in the objects, once that number exceeds a certain threshold. In contrast, the locus of the complexity effect on transformation and comparison rates, and on the time to reprocess on different trials, can be traced to the particular location of the bends. The implication is that not all parts of the objects are equally easy to process at different stages of the task. This, in turn, implies that not all parts have equal status in the representation. Thus, the parsing of an object does not take into account the location of a given piece, but once the representation has been set up, the location of the piece within the hierarchy is relevant for subsequent processing. However, instead of the principal axis's determining what is most accessible in the hierarchy, we have evidence that, for our stimuli, it is the structure at the ends of the figures that is more important. Below, we discuss two representational schemes in which this type of differential accessibility could exist. The first is a representation in which asymmetries about a top-bottom axis are represented along with asymmetries about a front-back axis, and the 
second is one in which the representation consists of nonredundant 2-D views of visible surfaces.

\section{GENERAL DISCUSSION}

Tarr and Pinker (1990) suggest that discriminating 2-D asymmetrical shapes requires discriminating between the two sides relative to the top-bottom axis. Therefore, asymmetrical 2-D patterns require a representation that specifies two dimensions simultaneously, such as a plane. We believe that this notion can be extended to the case of the 3-D objects in the present experiments, all of which were asymmetrical about the top-bottom axis. Thus, like Tarr and Pinker's (1990) 2-D objects, our 3-D objects require at least one 2-D view to keep track of the top-to-bottom ordering of parts and to discriminate between the sides of the objects relative to the top-bottom axis. However, the stimuli in the present experiments can be differentiated with respect to their sides relative to the top-bottom axis only as long as the bottom subarm is straight. This is true whether or not the central arm is bent, so, in this scheme, a central-arm bend does not add a level of complexity to the representation. In contrast, because the subarm bend was made relative to the front-back dimension, it does add an additional level of complexity, and it signals new information about the object. Thus, in this scheme, the subarm bend is a main contributor to the transformation time (i.e., slope of the RT function), because it implicates a second 2-D view (or plane) in the representation. There is other evidence that the distinction between the top-bottom and frontback axes is a useful one and that the two dimensions are differentially accessible (Franklin \& Tversky, 1990).

An alternative to representation in terms of front-back/ top-bottom axes has been proposed by Koenderink (Koenderink \& van Doorn, 1979). In this scheme, a representation consists of nonredundant views of the different possible visible surfaces of an object. Each view is a node in the representation, and the time to compare two 3-D shapes is a function of the number of nodes (i.e., intermediate views) represented between the nodes for the initial views of each shape. With respect to our stimuli, the bend in the main axis does not add an additional visible surface plane, whereas the $90^{\circ}$ bend in the subarm does create a new surface plane (and hence, an additional level of complexity). It is conceivable, in this approach, that some nodes (views) might be functionally more important than others; for example, some views might be more familiar or informative (Palmer, Rosch, \& Chase, 1981), depending on the task. Clearly, these two theoretical alternatives share some similarities that only further research will disambiguate.

It is likely that there are categories of objects that might require a different sort of representation than the two just proposed-for example, objects that do not have predominantly flat surfaces, objects whose axes are not joined at $90^{\circ}$ junctions, and objects that are curvilinear (e.g., Rock, Wheeler, \& Tudor, 1989; see also Koenderink, 1990). Research with such complex objects is sparse, but the studies that do exist have demonstrated that these sorts of objects are difficult to recognize from different views (Rock et al., 1989). This may mean that these objects are represented by structures that are difficult or impossible to align because the parts that are corresponding are difficult or impossible to determine.

To summarize, our data suggest that the ShepardMetzler objects we used are represented by sets of 2-D hierarchical representations. There are at least two possible representational schemes for deriving such representations. One is based on top-bottom/front-back axes (e.g., Tarr \& Pinker, 1990), and the other is a surfacebased representation (Koenderink \& van Doorn, 1979). We expect future experiments to differentiate between them.

\section{REFERENCES}

AUTODESK, INC. (1988). AutoCAD 10.0 [Computer program]. Sausalito, CA: Author.

Bethell-Fox, C. E., \& Shepard, R. N. (1988). Mental rotation: Effects of stimulus complexity and familiarity. Journal of Experimental Psychology: Human Perception \& Performance, 14, 12-23.

BIEDERMAN, I. (1987). Recognition-by-components: A theory of human image understanding. Psychological Review, 94, 115-147.

COOPER, L. A. (1975). Mental rotation of random two-dimensional shapes. Cognitive Psychology, 7, 20-43.

COOPER, L. A., \& PoDGorny, P. (1976). Mental transformations and visual comparison processes: Effects of complexity and similarity. Journal of Experimental Psychology: Human Perception \& Performance, 2, 503-514.

COOPER, L. A., \& ShePARD, R. N. (1973). Rotation of mental images. In W. G. Chase (Ed.), Visual information processing (pp. 75-176). New York: Academic Press.

FINLEY, G. (1985). A high-speed plotter for vision research. Vision Research, 25, 1993-1997.

FoLK, M. D., \& LUCE, R. D. (1987). Effects of stimulus complexity on mental rotation rate of polygons. Journal of Experimental Psychology: Human Perception \& Performance, 13, 395-404.

Franklin, N., \& TVersky, B. (1990). Searching imagined environments. Journal of Experimental Psychology: General, 119, 63-76.

Friedman, A., \& Hall, D. L. (1989, June). Effects of figural complexity, principal axis, and spatial ability on mental rotation performance. Poster presented at the meeting of the Canadian Psychological Association, Halifax, NS.

Friedman, A., \& HALL, D. L. (1992, July). The importance of being upright: The $Y$ axis advantage in mental rotation of $3 D$ objects. Paper presented at the 25th International Congress of Psychology, Brussels.

Friedman, A., \& Pilon, D. P. (in press). Effects of distance between objects and distance from the vertical axis on shape identity judgments. Memory \& Cognition.

Friedman, A., Pilon, D., \& Gabrys, G. L. (1988). Cognitive coordinate systems for mental rotation. Canadian Psychology, 29, 383.

Hinton, G. (1979). Some demonstrations of the effects of structural descriptions in mental imagery. Cognitive Science, 3, 231-250.

HochbERG, J., \& GelLMAN, L. (1977). The effect of landmark features on mental rotation times. Memory \& Cognition, 5, 23-26.

HofFman, D. D., \& RichaRds, W. A. (1984). Parts of recognition. Cognition, 18, 65-96.

HuMPHREY, G. K., \& Jolicoeur, P. (1988). Visual object identification: Some effects of image foreshortening and monocular depth cues. In Z. W. Pylyshyn (Ed.), Computational processes in human vision: An interdisciplinary perspective (pp. 430-444). Norwood, NJ: Ablex. 
Humphreys, G. W. (1984). Shape constancy: The effects of changing shape orientation and the effects of changing the position of focal features. Perception \& Psychophysics, 36, 50-64.

JolicoeUr, P., REgehr, S., SMITH, L. B. J. P., \& SMith, G. N. (1985). Mental rotation of representations of two-dimensional and threedimensional objects. Canadian Journal of Psvchologv, 39, 100-129.

Jolicoeur, P., Ullman, S., \& MaCkay, M. (1986). Curve tracing: A possible basic operation in the perception of basic routines. Memory \& Cognition, 14, 129-140.

Just, M. A., \& CARPENTER, P. A. (1976). Eye fixations and cognitive processes. Cognitive Psychology, 8, 440-480.

Just, M. A., \& Carpenter, P. A. (1985). Cognitive coordinate systems: Accounts of mental rotation and individual differences in spatial ability. Psychological Review, 92, 137-172.

Koenderink, J. J. (1990). Solid shape. Cambridge, MA: MIT Press.

Koenderink, J. J., \& Van Doorn, A. J. (1979). The internal representation of solid shape with respect to vision. Biological Cybernetics, 32, 211 -216.

Kosslyn, S. M. (1981). The medium and the message in mental imagery: A theory. Psychological Review, 88, 46-66.

Krueger, L. E. (1978). A theory of perceptual matching. Psychological Review, 85, 278-304.

KRUEGER, L. E. (1985). Effect of intermixed foveal and parafoveal presentation on same-different judgments: Evidence for a criterioninertia model. Perception \& Psychophysics, 37, 266-271.

MARR, D., \& NishihaRA, H. K. (1978). Representation and recognition of the spatial organization of three-dimensional shapes. Proceedings of the Royal Society of London: Series B, 200, 269-294.

METZLER, J., \& SHEPARD, R. N. (1974). Transformational studies of the internal representation of three-dimensional objects. In R. L. Solso (Ed.), Theories in cognitive psychology: The Loyola Symposium (pp. 147-201). Potomac, MD: Erlbaum.
Palmer, S. (1989). Reference frames and the perception of shape and orientation. In B. E. Shepp \& S. Ballesteros, (Eds.), Object perception (pp. 121-163). Hillsdale, NJ: Erlbaum.

Palmer, S., Rosch, E., \& Chase, P. (1981). Canonical perspective and the perception of objects. In J. B. Long \& A. D. Baddeley (Eds.), Attention and performance IX (pp. 135-151). Hillsdale, NJ: Erlbaum.

PARsons, L. M. (1987). Visual discrimination of abstract mirror-reflected three-dimensional objects at many orientations. Perception \& Psychophysics, 42, 49-59.

PYLYSHYN, Z. W. (1981). The imagery debate: Analogue media versus tacit knowledge. Psychological Review, 88, 16-45.

Rock. I. (1973). Orientation and form. New York: Academic Press.

Rock, I., WheEler, D., \& TUDOR, L. (1989). Can we imagine how objects look from other viewpoints? Cognitive Psychology, 21, 185210.

ShePARd, R. N., \& CoOper, L. A. (1982). Mental images and their transformations. Cambridge, MA: MIT Press.

Shepard, R. N., \& Metzler, J. (1971). Mental rotation of threedimensional objects. Science, 171, 701-703.

TARR, M. J., \& PINKER, S. (1990). When does human object recognition use a viewer-centered reference frame? Psychological Science, 1, 253-256.

Ullman, S. (1984). Visual routines. Cognition, 18, 97-159.

Ullman, S. (1989). Aligning pictorial descriptions: An approach to object recognition. Cognition, 32, 193-254.

YutLle, J. C., \& Steiger, J. H. (1982). Nonholistic processing in mental rotation: Some suggestive evidence. Perception \& Psychophysics, 31, 201-209.

(Manuscript received June 21, 1993;

revision accepted for publication February 16, 1994.) 